Fecha de recepción: abril 2020 Fecha de aceptación: mayo 2020 Versión final: junio 2020

\section{Resonancias de Bauhaus en Latinoamérica y el caso de la Escuela de Diseño en Cuenca, Ecuador Entre similitudes y diferencias, ¿una Bauhaus latinoamericana?}

Genoveva Malo ${ }^{(1)}$

Resumen: Este artículo busca contextualizar los momentos histórico-culturales de inicios y finales del siglo XX en los cuales se crearon, por un lado, la escuela alemana de Diseño Bauhaus y la escuela de Diseño en Cuenca, Ecuador. Nos interesa también reflexionar sobre las características que dieron forma a cada uno de estos proyectos académicos que tuvieron en común un fuerte énfasis en la artesanía y en la revalorización de la práctica del oficio en el proceso de enseñanza-aprendizaje del Diseño. Las similitudes y diferencias encontradas en la investigación marcan las características de las resonancias que Bauhaus pudo haber tenido en América Latina, con un contenido propio. Nos referimos a la problemática de la identidad y los posibles cruces con el Diseño y su construcción como parte de un proceso cultural.

Palabras clave: Relación Diseño-artesanía - Bauhaus y Latinoamérica - Diseño y contexto cultural - mundos del Diseño.

[Resúmenes en inglés y portugués en las páginas 103-104]

(1) Genoveva Malo, Ecuador. Profesora investigadora de la Universidad del Azuay, Cuenca. Diseñadora, Magister en Diseño. Sus investigaciones se enmarcan en la epistemología del diseño, los discursos del Dseño en el contexto contemporáneo con énfasis fuerte en el diseño vinculado a la cultura y artesanía.

Aunque la escuela de Diseño en Cuenca, Ecuador propusola valorización de la artesanía,al igual que la Bauhaus, pudo haber sido la más rupturista e innovadora con respecto a otras escuelas creadas en Latinoamérica de acuerdo al modelo europeo. 


\section{Diseño en Europa y Diseño en América Latina: el contexto del surgimiento de la disciplina, mundos distintos}

Es el Diseño, ante todo, un articulador y reflejo de tejidos sociales, humanos, culturales, históricos, tecnológicos y ambientales. La historia del surgimiento de la disciplina,su recorrido e intervenciones en la cultura material y simbólica pueden leerse en el marco de las trasformaciones de la sociedad y los contextos que generan, permiten y validan su existencia.

Como disciplina, el Diseño ha problematizado constantemente su relación con el arte, la artesanía y los modos productivos. Estas relaciones, tensiones e intersecciones han sido una constante en el recorrido del Diseño desde su nacimiento, y en su desarrollo y consolidación. Sin duda, han definido caminos para configurar y potenciar su accionar en el campo simbólico y productivo de las sociedades.

Intentamos en este ensayo recomponer las huellas que marcaron el impacto de la Bauhaus sobre la manera de pensar y hacer Diseño en América Latina y,concretamente,en Ecuador. De manera particular, nos interesa mirar nuestra propia historia regional para reconocer las marcas de identidad de un Diseño que surgió en Cuenca,entre similitudes y diferencias con la primera escuela de Diseño en el mundo y, así, dejar abiertas reflexiones sobre la coyuntura del Diseño en América Latina, sus impactos, sus huellas, su historia y su futuro. Desde la mirada del pensamiento complejo también proponemos encontrar las diferencias entre lo que aparentemente es igual (relación del Diseño y la artesanía en dos momentos de emergencia de la disciplina) y las similitudes entre lo que aparentemente es diferente (momentos culturales distintos), para así tejer las relaciones posibles. Como dice Tomás Maldonado (1977) "el único modo de conferir a la tradición Bauhaus un contenido vivo es contrarrestarlo con nuestro propio punto de vista contemporáneo" (p. 157). Este punto de vista contemporáneo, es sin duda, un pensamiento relacional que busca identificar y construir diversos vínculos de sentido.

Los inicios de la disciplina académica del Diseño, con la escuela Alemana Bauhaus en 1919, y su singular modelo pedagógico, definieron una relación muy fuerte con la artesanía y el reconocimiento del oficio como clave en la enseñanza del Diseño; aunque con el recorrido posterior de la escuela, en sus diferentes sedes y con sus diferentes directores, se hayan cedido estos esfuerzos inicial es hacia un marcado determinismo industrial. La huella de "lo industrial" en el modelo fue, tal vez, el trasplante más visible en las carreras universitarias de Diseño en Latinoamérica que, en su mayoría, adoptaron propuestas y enfoques curriculares de otra realidad contextual.

Es evidente que la emergencia de la disciplina, a inicios del siglo XX en Europa, a mediados de siglo en Estados Unidos y casi enseguida en América Latina, ocurre en situaciones de contexto social e histórico que, con las diferencias y particularidades de cada caso, habrían reflejado condiciones específicas. Sin embargo, es importante diferenciar los contextos: en la Alemania de fin de siglo XIX e inicios del XX, la creciente industrialización marcaba la urgencia de formar diseñadores que respondieran a la estética vinculada a un nuevo modelo productivo, visión que se consolidó fuertemente en Estados Unidos.

En América Latina, en general, el surgimiento del Diseño habría caracterizado un hecho que podría resultado paradójico: por un lado la profundización de un estado de depen- 
dencia, pues los modelos se importaban y, a la par, la urgente necesidad de transformación local y construcción de identidad, discursos que se hacían potentes después de la segunda mitad de siglo (Jaramillo, 1991).

Esta visión de un trasplante del modelo europeo con escasa relación a la realidad contextual acentúa la tensión centro-periferia y el urgente cambio social. Los orígenes y la consolidación de la profesión en cada país fueron distintos. En un grado de mayor, de menor o casi nula relación,se establecieron vínculos con el contexto, pues en su mayoría se trasplantaron modelos europeos con muy poca cercanía a la realidad de cada país o región y a su grado de desarrollo.

En países como Argentina, Chile y Brasil se puede ubicar al surgimiento de la disciplina alrededor de los años cincuenta,en el contexto de las políticas de sustitución de importaciones y la imperante necesidad, mas no consolidación, del desarrollo industrial (Fernandez \& Bonsiepe, 2008). El ideal modernizador marcaba una manera de entender al Diseño y la historia se escribió con ciertos acentos de especificidad local (Calvera, 2017).

En Cuenca, Ecuador, sesenta y cinco años más tarde de la creación de la emblemática Bauhaus, en 1984 surgió una propuesta académica sin precedentes en la región, que fue pionera en el país. Se planteaba la formación en Diseño desde una propuesta propia, distante de condicionamientos industriales que eran ajenos al entorno en donde se propuso esta innovadora escuela de Diseño. Esta carrera supuso, en ese momento, una muy estrecha vinculación con el contexto y, por ende, con los modos productivos artesanales que marcaban el momento histórico y cultural en América Latina (Cuenca, 1984).

El Diseño en Bauhaus y el Diseño en Cuenca son interesantes historias que se entrelazan por un tema común: la artesanía y su fuerte vínculo con el Diseño. Son historias que se cruzan y dan cuenta de la complejidad de relaciones que pueden establecerse en temporalidades distintas y en contextos geográficos y culturales diferentes del siglo XX. Pero, ¿hablamos de la misma relación con la artesanía? ¿hablamos de un enfoque compartido? En un primer momento de análisis, desde una mirada panorámica, podríamos encontrar un estrecho vínculo entre el modelo de enseñanza de Bauhaus en Weimar, Alemania de los años veinte del siglo anterior y la carrera de Diseño en Cuenca de los años ochenta, a manera de una resonancia que se hace evidente en el reconocimiento, valorización de la artesanía y el énfasis académico en el aprendizaje del oficio, la técnica y los procesos. Sin embargo, los contextos culturales e históricos a los que hacemos referencia son, sin duda, diferentes, y pertenecen a mundos diversos que refieren a condiciones de producción, a modos de pensamiento y momentos culturales muy distintos.

Por lo tanto, será preciso profundizar en los factores de contexto que marcaron las condiciones para el surgimiento del Diseño como disciplina en dos lugares diferentes y en dos tiempos distintos de un mismo siglo; así como queremos reflexionar sobre los modos de concebir un proceso académico de enseñanza para encontrarlas similitudes y diferencias en un enfoque caracterizado por una marcada presencia de la artesanía en cuanto a modo productivo y construcción simbólica.

Por lo expuesto, será importante comprender que ambos momentos culturales corresponden a dos visiones de mundo diferentes. Desde el enfoque de la filosofía de la ciencia, tomamos para este análisis el concepto de mundo extraído de la teoría de Nelson Goodman (1990) quien en su libro Maneras de hacer mundos enfatiza en la posibilidad de 
comprensión de la existencia de diversos sistemas simbólicos, tanto en las ciencias, como en las artes, la filosofía y en los discursos de la vida cotidiana, que conforman mundos diferentes. En este sentido, interpretamos cada momento cultural como un mundo creado por símbolos que el hombre construye como ser cultural.

Señala Goodman (1990), "no hablamos ahora de múltiples alternativas posibles a un único mundo real, sino, por el contrario, de múltiples mundos reales" (p. 19). A partir de esta reflexión, queremos identificar la configuración de mundos en la relación con el Diseño académico y la artesanía en dos momentos diferentes de la historia, a manera de construcción de mundos diversos y, a su vez, nos interesa indagar en las interrelaciones posibles entre estas diferentes versiones de mundo, a manera de tejidos complejos. Para el autor, la clave está en la comprensión de la existencia de una:

Pluralidad de versiones correctas, que no son irreductibles a una sola y que entran en mutuo contraste, no deberemos buscar su unidad tanto en un algo, ambivalente o neutral, que subyace a tales versiones cuanto en un organización global que las pueda abarcar a todas ellas (Goodman, 1990, pp. 22-23).

El marco de referencia cobra importancia, según Goodman (1990) pues éste refiere a los sistemas de descripción más que a aquello que estos describen.

Si nos preguntamos cómo es el mundo se nos puede responder describiéndolo bajo uno o bajo varios de esos marcos de referencia, pero ¿Qué se nos podría contestar si insistiésemos en preguntar cómo habría de ser el mundo si dejáramos al margen cualquier marco de referencia? (p. 19).

Nuestros marcos de referencia son así los momentos histórico culturales y el modo cómo los leemos, desde una mirada de relaciones complejas. Así, nos proponemos inicialmente conocer las condiciones de contexto que antecedieron y estaban presentes en el surgimiento de la primera escuela de Diseño en el mundo y de la primera escuela de Diseño en Ecuador.

\section{Condiciones de contexto en el surgimiento del Diseño en Bauhaus y en Cuenca}

En un breve análisis histórico, recordemos que el estado alemán no existió sino hasta finales del siglo XIX y, de alguna manera, la influencia prusiana marcaría la tendencia de la escuela. Si bien no se comprometía con fines políticos, sí tenía un énfasis especial sobre un ideal democratizador y socialista que miraba a un horizonte moderno.

A nivel constructivo, los inicios del siglo XX marcaron, un escenario polarizado: en la arquitectura predominaba el eclecticismo historicista y, por otro lado, empezaba a haber cierto desarrollo, a manera de conurbaciones por la influencia de las fábricas de acero en pleno auge de crecimiento y desarrollo. Dos realidades antagónicas, entre ingeniería y 
arquitectura, demandaban en Alemania la necesidad de buscar modelos que sean capaces de homogeneizar la producción en un solo modelo (Hervás y Heras, 2015).

En este sentido, no resultaría casual que Walter Gropius haya integrado inicialmente la directiva de lo que pudo ser la antecesora de la Bauhaus: la Werkbundo Asociación Alemana para la Industria. El proceso industrial avanzaba a pasos agigantados y las llamadas artes aplicadas y la arquitectura requerían respuestas imperiosas.

Es indudable que eran tiempos en donde una sociedad burguesa en amplio crecimiento tenía más acceso al arte aplicado. Estaba latente y urgía, en este contexto, conciliar la creciente industria y las artes, una dicotomía potente en el contexto del surgimiento del Diseño como disciplina en Alemania de inicios de siglo. Los objetivos eran claros: la artesanía ofrecería lo mejor de su conocimiento en favor de una construcción total, desde los objetos más simples hasta la arquitectura. Parecería así que se estaría poniendo a la artesanía y su bagaje de conocimiento al servicio de la producción industrial.

En este contexto, una propuesta curricular innovadora marcaría el devenir de una institución que, más que una escuela de Diseño, se convertiría en el espacio más provocador, reflexivo y activo en el campo del Arte, el Diseño y la Arquitectura, pues convocó a las más diversas expresiones del arte y la producción.

Las influencias, antecedentes y el contexto del surgimiento de esta mítica escuela tienen diversos orígenes como los postulados de John Ruskin y el movimiento Arts and Crafts, que, desde la postura de reivindicación de la artesanía, ya había cuestionado la relación de las artes en relación a la industria. Así, la Bauhaus catalizaría,desde una visión socialista y ética, una reconciliación entre arte y técnica que se habían fragmentado a partir de la revolución industrial. Esto, sin duda, caracterizó un Diseño cuya fundamental preocupación estaría en la relación forma -función- producción, sentando de esta manera, las bases para el diseño moderno que alcanzó su madurez con la escuela HfG ULM.

Este era el ambiente político y social de fin de siglo XIX que, junto al escenario de comienzos de un nuevo siglo, marcado por la guerra y posguerra, la inflación, la pobreza y los disturbios, se convertiría en el espacio para el florecimiento de una nueva propuesta de reconciliación entre las formas de expresión del arte aplicado en muchas de sus manifestaciones y la producción.

En estas condiciones, nos preguntamos: ¿cuáles eran los postulados sobre la enseñanza que hicieron que este legendario centro de Diseño tenga una profunda influencia social y cultural? ¿cuál ha sido su principal contribución al mundo del Diseño? ¿cuál fue el contexto que caracterizó el surgimiento de la escuela Bauhaus? ¿qué rol tenía el aprendizaje del oficio y la relación con las artesanías? ¿qué resonancias pudo tener en América Latina? En palabras de Tomás Maldonado (1977), Bauhaus fue, más que una realidad, una fábula; hecho que no es alarmante, sino que da cuenta de la complejidad y riqueza de la propia realidad. Sus postulados de enseñanza y las características más importantes de su didáctica estuvieron sin duda en la praxis manual y artística, el desarrollo de una personalidad activa y la ejercitación de los sentidos. Es decir, se concebía al aprendizaje no como una actividad exclusiva del intelecto sino también de las emociones: el Diseño total.

Bauhaus, sin duda, fue no solo un centro de enseñanza del Diseño sino un centro de experimentación pedagógica que supuso un impacto en la gráfica, los textiles, la industria 
y, posteriormente, la arquitectura moderna; a partir de códigos visuales y didácticos muy potentes que hoy siguen dando forma a nuestra idea de Diseño, Arte y Arquitectura.

Los postulados más importantes de Bauhaus tenían que ver con la construcción que, como idea fundamental de creación, marcaba una forma de enseñar y aprender el Diseño desde la eficiencia funcional y formal. En ese sentido, las formas simples, los colores primarios, la estandarización como premisa, la utilización de materiales industriales, marcarían una manera de concebir la forma orientada a la producción de diseño industrial.

Los principios fundamentales de Bauhaus, fueron: el aprender haciendo, la recuperación del saber artesanal como esencial en la práctica del Diseño, el uso de formas simples, colores primarios, la funcionalidad como objetivo, la construcción como idea fundamental de creación, la arquitectura como espacio ideal, la estandarización y el diseño como hecho social. Se buscaba formar al artesano ideal, al diseñador ideal y arquitecto ideal. Sin duda esta escuela estableció los criterios del diseñador moderno sobre las bases y tendencias de la funcionalidad como valor irrenunciable.

¿Sería esta acaso la formación de un artesano ideal?, es una de las preguntas que se han hecho en torno a la escuela y que dan cuenta de la necesidad de explicar el vínculo con el aprendizaje de la artesanía y el oficio en los talleres de formación básica, tal como lo había dicho Walter Gropius, primer director de Bauhaus en el manifiesto fundacional: “....arquitectos, escultores, pintores, todos debemos volver a la artesanía..." (Manifiesto de la Bauhaus, 1919). Así, se había propuesto un modelo pedagógico que promovía el acercamiento a la artesanía y a los oficios como un proceso de comprensión de modos productivos que posteriormente podrían ser llevados a la industria. Se sostenía que industria y artesanía podrían acercarse de un modo paulatino

De esta manera, Bauhaus buscaba, en los oficios artesanales y el arte, una reconciliación con técnica (relación fragmentada a partir de la Revolución Industrial), aunque este objetivo inicial, paradójicamente se iría alejando poco a poco de la artesanía hasta ceder a las demandas de una estética industrial.

El legendario centro de estudios fue un espacio pedagógico que supuso un impacto sin igual en el diseño grafico e industrial del siglo XX. Se desarrolló un código visual y didáctico tan potente, que lo que inició como un centro de arte y artesanía, luego se convirtió en una institución que marcó fuertemente la relación forma función en el diseño y la arquitectura. Atrajo a muchos jóvenes que pensaban que otro mundo era posible. Cien años después, seguimos reflexionando sobre las repercusiones e impactos de este legendario centro de estudios.

Bauhaus constituyó un periodo de esplendor en el Diseño sin igual. Las intersecciones entre el arte, el diseño y la arquitectura eran el reflejo de una amalgama de ideas, corrientes y personajes diversos. Fue definitivamente un centro de vanguardia que tuvo tres sedes: Weimar, Desau, y Berlin, que vio su cierre en manos del ejército Nazi. Fueron Gropius, Meyer y Mies Van De Rohe sus directores.

Cada etapa, con estilos y enfoques diferentes, constituyó sin duda un referente para el diseño industrial, textil, gráfico, tipográfico, interiorismo y arquitectura. Con sus talleres de textiles, metales, fotografía, arte, madera, las fiestas las danzas y la vida misma en Bauhaus, esta se convirtió en una de las escuelas más productivas y criticadas también de su tiempo. 
El concepto de Diseño total estaba implícito. Tomás Maldonado (1977) había escrito: "educar por el arte, la acción y el trabajo son las constantes que pueden entresacarse del pensamiento pedagógico de los maestros de la Bauhaus" (p. 150) y de alguna manera, esto refleja un sistema pedagógico que trascendió el aula y se convirtió en una forma de vida. Bauhaus fue cerrada en manos del gobierno nazi en 1933, pero su huella estuvo presente y pronto las repercusiones se sentirían en América, primero en Estados Unidos y luego en Latinoamérica.

\section{¿Una Bauhaus latinoamericana?}

La historia y postulados de Bauhaus se cruzan con Cuenca, que hace 35 años vio nacer a la primera escuela de Diseño. Una Escuela que, al igual que su antecesora, convocó a artistas, arquitectos y artesanos a la formación de diseñadores. Se equiparon talleres de joyería, cerámica, textiles y madera para que la práctica y el aprendizaje del oficio sea un factor fundamental en la relación con el contexto, la identidad y el sistema productivo de la región (Cuenca, 1984).

El contexto de referencia histórico cultural nos ubica alrededor de los años setenta, como década precedente a la creación de esta carrera de Diseño. Es importante mencionar que el ambiente político cultural en la región estaba marcado por la salida de dictaduras en varios países de América del Sur. Ecuador vivió tres dictaduras: una civil y dos militares. Como reacción a esa situación, en toda América se dieron vientos de recuperación de la tradición, de lo propio, de lo latinoamericano como búsqueda de una identidad emancipadora frente a situaciones de represión. Podría decirse que era una ola que recorría América con propósitos de reivindicación, tradición, reparación.

En toda la región había vientos de Latinoamericanismos, se buscaba la recuperación de lo propio, de la tradición. Por otro lado, se vivía también lo que podría decirse la primera presencia fuerte de la literatura ecuatoriana en el mundo, la época de lo latinoamericano. Octavio Paz en la literatura y la música social marcaba el ambiente de la década, música que recuperaba también la tradición y valores populares. El feísmo de Cuevas, surgido desde México recorría toda América. En el caso de Ecuador, el Arte llamado precolombino o el precolombinismo era la presencia fuerte en las artes plásticas y posiblemente la primera presencia del arte ecuatoriano en el mundo a través de Guayasamin (Jaramillo, 2019).

En este escenario, este mundo, podemos reconocer que si hay algo que subyace a la propia creación de la carrera son las condiciones del contexto: los referentes histórico culturales que marcan fuertemente la construcción cultural y la producción simbólica y material de época, que sin duda darían sentido al nacimiento de la carrera de Diseño,por lo que sucedía en Latinoamérica. En este escenario, no resulta casual que un antropólogo haya sido quien propuso inicialmente la carrera de Diseño, pues la mirada desde la antropo- 
logía permitió una reflexión y análisis más profundo sobre lo que estaba sucediendo en América Latina.

En la década de los setenta se había fundado el CIDAP Centro de Artesanías y Artes Populares, acontecimiento que definió una relación importante con la artesanía que tendría el Diseño que surgió en Cuenca; pues poco tiempo después, en 1984, en la actual Universidad del Azuay, el director Académico, Claudio Malo, director además de la mencionada institución de promoción y difusión de las artesanías en América, propuso la creación de una escuela de Diseño, en el marco de una fuerte relación con los valores culturales y productivos de la región. "El reto se planteaba en términos de cómo resignificar y decodificar al Diseño que sin duda tenía un enmarcamiento en la cultura industrial europea presente en la Bauhaus" (Jaramillo, 2019, p. 2).

Ese era el contexto en donde lo artesanal estaba presente, en donde la tradición se hacía evidente, un contexto en donde el enfoque de recuperación y revalorización se posicionaba en las expresiones de arte y cultura. En este escenario, en este mundo, se propusieron los retos de la creación de la Facultad de Diseño

La Universidad convocó para la creación de la carrera,a un grupo de arquitectos que estaban vinculados al arte y a la artesanía, en una evidente similitud con la Bauhaus. "Convocamos a profesionales de la arquitectura, artesanos, artesanas y artistas para fundar la primera escuela de Diseño, nos interesaba contar profesionales que tengan cierta inclinación por el arte y aprecio por la cultura local” (Malo, 2018, p. 3)

Dora Giordano, quien fue convocada unos pocos meses después para acompañar en la formulación del plan académico recuerda:

En los años ochenta, me llaman y me pregunto, ¿me convocan para una Bauhaus latinoamericana?, inicialmente fue una emoción muy fuerte, pero duró segundos, pues no podría haber una Bauhaus latinoamericana porque los hechos son irrepetibles y contextos no eran los mismos. Porque esta escuela iba a ser reivindicatoria de las tradiciones que habían surgido en el contexto de la Bauhaus. Entonces, ¿¿surgió la pregunta? qué carácter iba va a tener esta escuela de Diseño, el primer interrogante que surgió fue: ¿un trasplante? No. "Si apenas estamos Ilusoriamente industrializados como diría Galeano" (Giordano, 2019, p. 2)

Ese encuentro con “otra realidad” sería, según Giordano (2007), un detonante para construir una propuesta capaz de romper los modelos, hasta entonces establecidos, para las carreras de Diseño Industrial en Europa y posteriormente en algunos países de América Latina.

Allá, por los años ochenta, llegué a Cuenca por primera vez; me fascinó esta ciudad; imaginé entonces que sería posible descubrir ese sentido "latente", mentado en tantos discursos referidos a la identidad latinoamericana:

En el momento de primera aproximación a la ciudad, sólo hubo sensaciones; era una especie de encantamiento, sin dedicación de análisis racional alguno. 
Después... sucedieron vivencias, aquellas que irían hilvanando un proceso de conocimiento más profundo desde la subjetividad, es decir, desde una mirada intencional que busca y encuentra rasgos, elementos, relaciones...

Un contexto natural que enmarca y penetra el hábitat urbano, el ambiente, la arquitectura y los objetos se presentaba a través de una maravillosa conjugación de formas y colores. Todo parecía ofrecer certezas de lo genuino, en términos de construcción cultural (p. 27).

Diego Jaramillo, primer director de la carrera, sostiene que la carrera se propuso en un escenario de relaciones construidas con el contexto, había conciencia del estado de desarrollo en los sistemas productivos. La relación con la artesanía se mostraba potente para el desarrollo local y el fortalecimiento de la cultura. En este escenario, se imaginó una carrera de Diseño basada en tres enfoques fundamentales que daban forma a la propuesta curricular y a la ideología de la escuela. Estos tres pilares que configuraban el carácter de la carrera eran: el Diseño como lenguaje, el Diseño como práctica de transformación y el Diseño como hecho contextualizado. Como eje fundamental conceptual, se construiría el discurso del Diseño en referencia a la problemática de la morfología y la significación (Jaramillo, 1991).

La carrera de Diseño, creada en 1984 y pionera en el país, con su construcción curricular y enfoque genuino, abrió y recorrió caminos que le permitieron consolidar una oferta académica sólida. Aquel origen de un Diseño con enfoque integral, fuertemente vinculado a la prolífera y diversa producción artesanal de la región, con énfasis en diseño gráfico y de objetos fue abriéndose paso hacia nuevos campos de intervención, de la mano de los avances tecnológicos y de las nuevas maneras de pensar y hacer diseño.

La carrera fue concebida por un equipo de profesores que profundizó en la relación teoría-práctica y convocó a artesanos y técnicos del medio local en las ramas de carpintería, joyería, cerámica y textilería para crear espacios de enseñanza-aprendizaje basados en el conocimiento y exploración formal de las técnicas artesanales representativas en la región; sin duda una conexión con la inicial idea de la emblemática Bauhaus.

Así, en ese mundo construido por símbolos, referentes y condiciones de contexto no cabía el trasplante, pero sí la referencia a un modelo pedagógico innovador. Al decir de los fundadores, hubo, en la primera etapa, una clara conciencia de lo que significaba ese momento histórico cultural, las condiciones de producción eran diferentes.

El reto de la escuela de Diseño en Cuenca fue alejarla del signo de la industrialización, pues Ecuador no era un país industrializado, Cuenca no era una ciudad industrializada,pero sí era rica en artesanías y tradiciones. Este fue el desafío que marcó la personalidad de la escuela de Diseño en Cuenca: una construcción disciplinar desde el enfoque del contexto cultural y pertinencia local, una respuesta académica potente. La propuesta fue pensar al Diseño como parte de un proceso creativo en respuesta a una necesidad cultural (Giordano, 2019).

El desafío constituyó en proponer un modelo pedagógico con énfasis en la construcción del Diseño como hecho cultural y el Diseño como lenguaje. Posturas que evidenciaron el enfoque de los talleres, los cursos básicos de Diseño y Lenguaje y la posibilidad de resignificar los objetos. 
El estudio y producción de tipologías, más allá de la noción de tipo, como identificación de características para ser repetida en serie, fuertemente marcada en el pensamiento racionalista, definirían un enfoque de Diseño que buscó otras formas de expresión y modos de producción. Se presentaba así una oportunidad para la reivindicación de los valores propios a través de los procesos de seriación y tipologías, producción de formas que resignificaban objetos y procesos tradicionales. El énfasis estuvo, de acuerdo a los profesores fundadores, en la resignificación de la forma y la producción con un cambio de modelo propio de una producción orientada a la industria.

\section{A manera de reflexión final}

El análisis dedos mundos del Diseño y la artesanía analizados en el marco del surgimiento de la disciplina en Alemania y en Ecuador revelan una profunda conexión en lo que refiere ambas se vinculan fuertemente con su propio marco de referencia histórico cultural. La relación con la artesanía da cuenta de una constante en la disciplina que problematiza los modos productivos y simbólicos en cada caso.

La relación Diseño-artesanía ha cambiado, se modifica y transforma constantemente y este análisis da cuenta de un hecho constante en los procesos de construcción disciplinar. Las similitudes y diferencias encontradas en dos momentos culturales del Diseño revelan el carácter complejo de la disciplina.

Resulta interesante analizar cómo, un siglo después, algunos postulados de ambas escuelas mantienen vigencia absoluta: el Diseño como hecho social, como acto político, con propósitos éticos y estéticos, y el surgimiento de una nueva cultura del Diseño en donde confluyen todas estas expresiones. En el caso de Bauhaus,nos referimos a propósitos éticos y estéticos que crearon uno de los estilos más modernos, coherentes y reconocibles en todo el siglo XX, cuando el Diseño se volvió un asunto total. Para Cuenca,señalamos los propósitos éticos y estéticos en el marco de la construcción de sentido en el Diseño como ética cultural y su relación con la identidad. Las relaciones con la artesanía son así espacios de complejidad que se construyen entre similitudes y diferencias.

Por último, es necesario destacar la vigencia de ambos modelos en el mundo contemporáneo: el diseño como hecho total, integrador, interdisciplinar, propuesto por Bauhaus, y, por otro lado, el Diseño en relación a la problemática social y cultural tan presente en discusiones actuales y en la construcción de nuevas epistemologías.

\section{Referencias bibliográficas}

Bonsiepe, G. (1982). El diseño de la Periferia. Barcelona, España: Editorial Gustavo Gili. Calvera, A. (2017). Los tres orígenes del Diseño. Recuperado el Mayo de 2019, de http:// www.fadu.edu.uy/estetica-diseno-ii/files/2017/03/anna-calvera-tres-origenes-deldise\%C3\%B1o.pdf 
Cuenca, P. s. (31 de julio de 1984). Actas Pontificia Universidad Católica, Sede en Cuenca. Acta Consejo Académico. Cuenca.

De Ponti, J. \& Gaudio, A. (2008). Argentina 1940-1983. En S. Fernandez, \& B. Gui, Historia del Diseño en América Latina y el Caribe. Sao Paulo, Brasil: Blücher.

Fernandez, S. \& Bonsiepe, G. (2008). Historia del Diseño en América Latina. Sao Paulo, Brasil: Editorial Blucher.

Giordano, D. (18 de 11 de 2019). Intersecciones Bauhaus-escuela de Diseño en Cuenca. (G. Malo, Entrevistador).

Giordano, D. (2007). Las artesanías de Cuenca en el mundo contemporáneo. Artesanías de América, 27-40.

Goodman, N. (1990). Maneras de hacer mundos. Madrid: Gráficas Rogar.

Hervás y Heras, J. (2015). Las mujeres en la Bauhaus, de lo bidimensional al espacio total. Buenos Aires: Bibliográfica de Voros.

Jaramillo, D. (1991). Enseñanza del diseño y las artesanías. En CIDAP, Artesanos y Diseñadores. Cuenca: Ediciones CIDAP.

Jaramillo, D. (2019). Intersecciones Bauhaus-Escuela de Diseño en Cuenca. (G. Malo, Entrevistador) Cuenca.

Kentgns-Craig, M. (2001). The Bauhaus and América. Massachusetts: Bauhaus, Dessau Fundation.

Leon, E. \& Montore, M. (2008). Brasil. En S. Fernandez \& G. Bonsieppe, Historia del Diseño en América Latina y el Caribe. Sao Paulo, Brasil: Blücher.

Maldonado, T. (1977). Vanguardia y racionalidad: artículos, ensayos y otros escritos. Barcelona: Editorial Gustavo Gili.

Malo, C. (17 de 5 de 2018). Relación Diseño Artesanía.

\begin{abstract}
This article seeks to contextualize the historic-cultural period of the early and late twentieth century, in which the German Bauhaus School of Design and the School of Design in Cuenca, Ecuador were founded. The point of interest consists in analyzing the defining characteristics of each academic project, that share a common emphasis on artisanal crafts and the re-evaluation of it's practice in the teaching-learning process of design. The similarities and differences found in research signal the possible influence that Bauhaus may have had in Latin America. We also refer to the identity issue at its intersections with design and its construction as part of a cultural process.
\end{abstract}

Keywords: Design and artisanal crafts - Bauhaus and Latin America - Design and cultural context.

Resumo: Este artigo busca contextualizar os momentos histórico-culturais do início e do final do século XX, nos quais, foram criadas a Escola de Design alemã Bauhaus e a Escola de Design em Cuenca, Equador. Também estamos interessados em refletir sobre as características que moldaram cada um desses projetos acadêmicos que tinham em comum uma 
forte ênfase no artesanato e na reavaliação do trabalho no processo de ensino-aprendizagem do Design. As semelhanças e diferenças encontradas na pesquisa marcam as características das ressonâncias que Bauhaus pode ter tido na América Latina, com conteúdo próprio. Nos referimos a temática da identidade e possíveis interseções com o Design e sua construção como parte de um processo cultural.

Palavras chave: Relação Design-Artesanato - Bauhaus e América Latina - Design e contexto cultural - mundos do Design.

[Las traducciones de los abstracts fueron supervisadas por el autor de cada artículo] 